\title{
SOME UPDATES ON THE ROLE OF MAGNETIC FIELDS IN CATACLYSMIC VARIABLES
}

\author{
Coel Hellier ${ }^{1}$ \\ RESUMEN
}

En este resumen se cubren algunos aspectos de los recientes desarrollos en la comprensión del papel que juegan los campos magnéticos en variables cataclísmicas: presento una discusión sobre los recientes modelos de unificación DNO QPO; los límites de la magnetosfera del disco; algunas cuestiones relacionadas con la componente suave de cuerpo negro y la naturaleza de los espectros de rayos X en MCVs; si las estellas SW Sex son magnéticas; y finalmente, menciono el raro comportamiento de FS Aur y HS 2331+3905.

\section{ABSTRACT}

In this review talk I cover some recent developments in understanding the role that magnetic fields play in cataclysmic variables. I discuss the recent DNO-QPO unification models; the disk-magnetosphere boundary; some issues concerning the soft blackbody component and the nature of the X-ray spectra in MCVs; whether the SW Sex stars are magnetic, and finally I mention the weird behavior of FS Aur and HS $2331+3905$.

\section{Key Words: ACCRETION, ACCRETION DISKS - NOVAE, CATACLYSMIC VARIABLES}

\section{DNO-QPO UNIFICATION}

A major advance of recent years is a new understanding of the dwarf-nova oscillations (DNOs) and quasi-periodic oscillations (QPOs) seen in dwarf novae in outburst. Such things have been recorded and studied since the 1970 s, but only recently, with a series of papers by Warner and Woudt, do we have a compelling account of their origin (Woudt \& Warner 2002; Warner \& Woudt 2002; Warner, Woudt \& Pretorius 2003).

The heart of the model is a suggestion that, during DN outbursts, an equatorial belt of the white dwarf is spun up by enhanced accretion. The belt sliding over the white-dwarf core results in a dynamo, amplifying a seed field to the point where the field controls the accretion flow near the white dwarf by carving out a magnetosphere (Figure 1).

The standard DNOs are simply pulsations at the rotation period of this magnetosphere. The transience of the magnetosphere explains why the DNOs aren't seen in quiescence, while the low moment of inertia of the belt explains the low coherence of the oscillations - both of which had previously been problems for a magnetic explanation of DNOs.

The second element of the model is the idea that the magnetic ficld, playing on the inner edge of the disc, excites slow-moving waves which run prograde round the disk with a period $\approx 15$ times the magnetospheric spin period. These bulges modulate the light by simple obscuration, resulting in QPOs with

\footnotetext{
${ }^{1}$ Keele University, UK.
}

a characteristic $P_{\mathrm{QPO}} \approx 15 P_{\mathrm{DNO}}$.

A second type of DNO then results from reprocessing of the first DNO off the QPO bulges, giving the beat relation $1 / P_{\mathrm{DNO} 2}=1 / P_{\mathrm{DNO} 1}-1 / P_{\mathrm{QPO}}$. Further, Warner et al. (2003) claim a third type of DNO, which they suggest results from the rotation of a field attached to the body of the white dwarf, not the spun-up belt. This is rotating more slowly and so produces 'long period DNOs'. All of this interpretation is supported by an impressive amount of observational documentation.

The above theory raises the question of whether the same QPO bulges are excited at the inner edge of disks in intermediate polars. Certainly, such bulges are the most plausible explanation for the 5000-s QPOs seen in GK Per during outburst (e.g. Hellier, Harmer \& Beardmore 2004), but such things are not generally reported in IPs in quiescence. One reason might be observational: with a typical 1000-s spin period, the QPO period would be $\sim 4 \mathrm{hrs}$, and it is hard to observe for the dozen cycles that would be needed to prove the presence of a low-level, incoherent modulation. Further, such signals might be masked by orbital-cycle variations.

A second explanation might be that the bulges are only excited when there is strong slippage between the magnetic field and the inner disk, and that this isn't so in intermediate polars in 'heir equilibrium, quiescent state - a topic that seems worth pursuing observationally.

An interesting point (noticed by Warner \& 


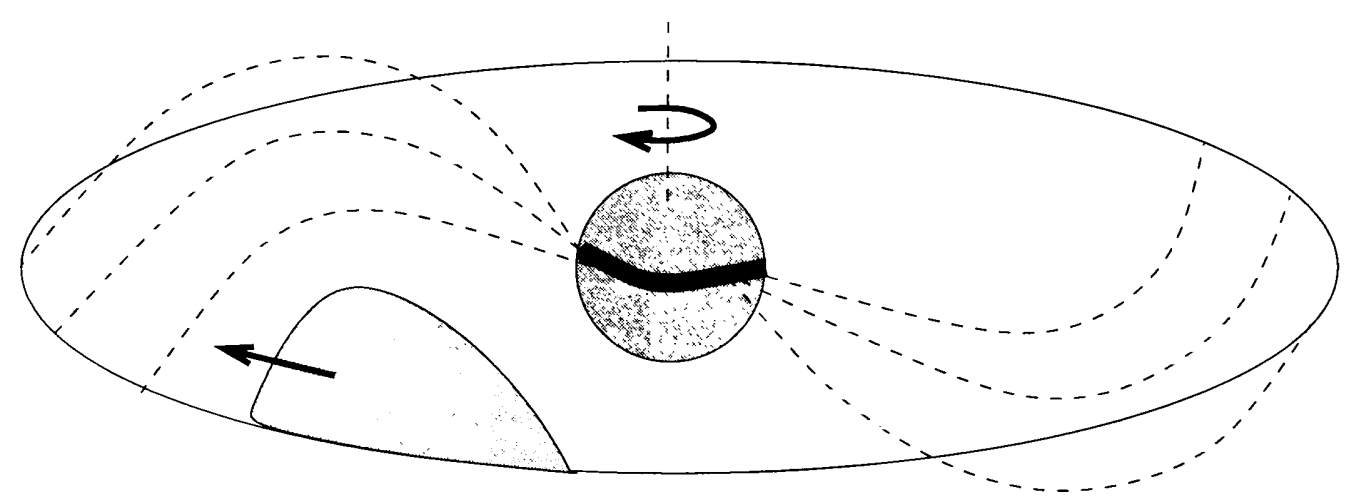

Fig. 1. A schematic of Warner \& Woudt's DNO/QPO model, with dynamo action in a spun-up belt producing a transient magnetosphere, which excites travelling waves on the inner edge of the disk.

Woudt 2002 and independently by Mauche 2002) is the fact that the QPO/DNO ratio can be extended over 5 orders of magnitude to cover the much faster QPOs seen in neutron-star and black-hole binaries. Is this coincidence or does it imply a causal similarity? There is valid skepticism over any link, since of course black-holes don't have a surface, nor a permanent magnetic field, and thus are unlikely counterparts of white dwarfs. However, in the model above the field is also a transient one created by a dynamo, and the action occurs at the interaction of the field with the inner disk, with the solid surface playing little role. Thus the situations are not as dissimilar as they may at first appear.

\section{THE DISK-MAGNETOSPHERE BOUNDARY}

While on the subject of the disk-magnetosphere boundary, it bears restating that this is one of the least understood regions of a CV. A paper on FO Aqr by Evans et al. (2004) shows that the accretion curtain appears to be swept back, trailing the magnetic pole by a quarter of a cycle. The opposite was found in PQ Gem (Mason 1997), where the accreting field lines lead the pole. One can then ask whether these twists are related to disk-field disequilibrium and thus to the torques on the white dwarf. At first sight it appears so, since the white dwarf in $\mathrm{PQ}$ Gem is spinning down whereas that in FO Aqr is currently spinning up. However, FO Aqr has changed from a period of spin-down to one of spin-up, without any obviously related change in the spin-pulse profile. Thus any interpretation is problematic, and the whole issue of the disk field interaction and the resulting torques is one that could do with more study.

\section{THE FOOTPRINT}

Turning now to the accretion footprint on the white dwarf, Ramsay \& Cropper (2004) have proposed a major re-evaluation of the accretion process in AM Her stars. For two decades it has been conventional wisdom that the AM Her stars show a strong 'soft excess' over that expected in the simplest accretion model (a hard-X-ray-emitting shock which irradiates the white-dwarf surface, resulting in soft blackbody emission that amounts to half the total flux). The excess is usually attributed to 'blobby accretion' in which blobs of material do not shock, but penetrate the white-dwarf surface and thermalize, greatly boosting the soft/hard ratio.

Now, from a systematic analysis of XMM data, Ramsay \& Cropper (2004) find that most AM Her spectra are indeed compatible with the simple model, and that previous reports of soft excesses were, to a large extent, artefacts of calibration and band-pass uncertainties (Figure 2).

However, a small number of systems do show a large soft excess, and are presumably dominated by blobby accretion. But why? Ramsay \& Cropper discuss the obvious variables such as field strength. but find no obvious correlation with the presence of a soft excess.

A similar question arises in the intermediate polars. Since Rosat (e.g. Haberl \& Motch 1995) we've known of a minority of IPs that show a softblackbody component, but the majority do not. Again, we have no good explanation for the difference, and no obvious correlation with field strength or other variables to guide us.

V405 Aur is one of the IPs with soft blackbody emission, and is also peculiar in that it shows a single-humped spin pulse at hard-X-ray energies but a double-humped pulse at softer energies. One idea explains the difference between single-humped and double-humped IPs as an absorption effect: IPs with short, fat accretion columns beam X-rays upwards and so produce double-humped pulsations, whereas IPs with tall, thin columns beam X-rays sidewars and produce single-humped pulsations (e.g. Hellier 


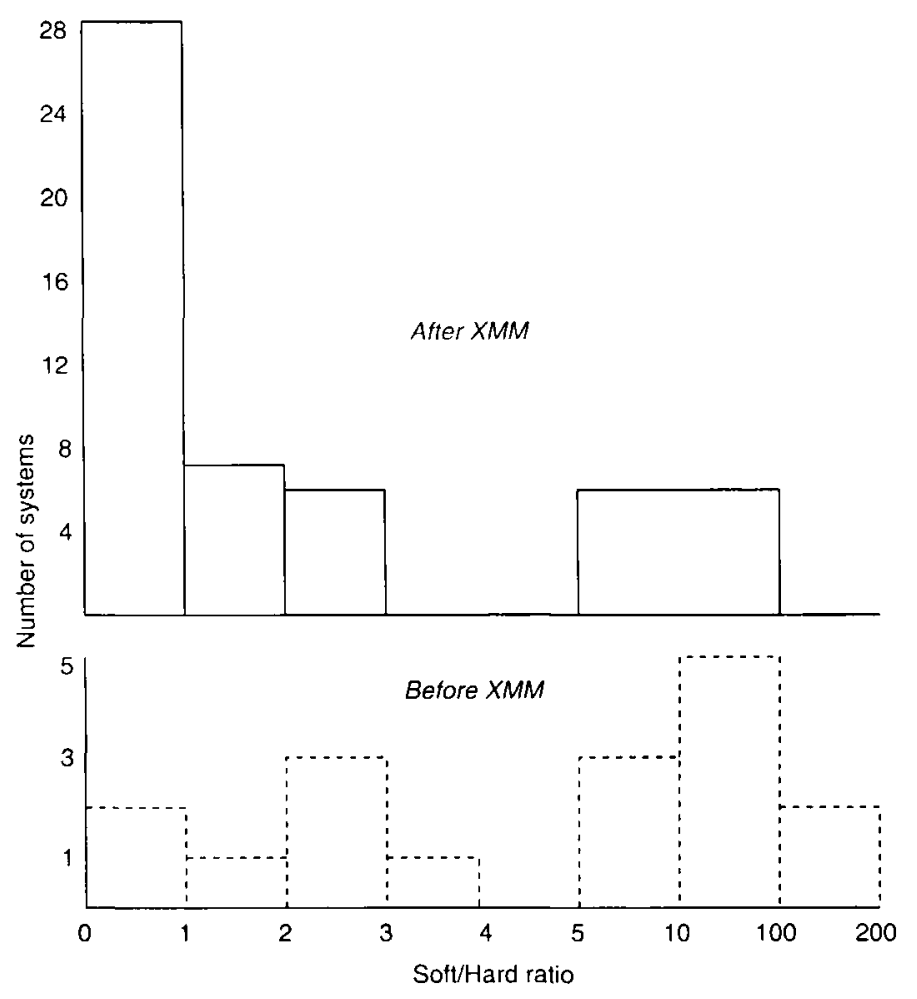

Fig. 2. Rarnsay and Cropper's reassessment of the energy balance in AM Her stars. Before XMM (bottom) it was thought that most AM Hers showed a strong soft excess. Now (top) only a minority show a soft excess, and the majority are compatible with the standard model.

1995; Allan (t al. 1996).

However, an analysis of XMM data by Evans \& Hellier (2004) shows that, at least in V405 Aur, the double-humped soft pulse is not the product of absorption. Instead it is a modulation of the visible area of the blackbody component, resulting primarily from variable foreshortening of the heated polecaps as the white dwarf rotates. It remains to be seen what this implies for the double-humped optical pulsation, and whether the findings are applicable to other double-humped IPs.

\section{THE X-RAY SPECTRA}

The standard model for X-ray spectra in IPs invokes a stratified column in which matcrial cools beneath the accretion shock. By assuming optically thin, collisionally ionized emission, onc can use a code such as MEKAL and sum the emission botwern the hot shock and the point where the columm becomes optically thick as it merges with the white dwarf. Such a model gives an excrllent representation of the spectrum of EX Hya (Cropper et al. 2002).

However, Mukai et al. (2003) report that EX Hya is unusual. From an analysis of Chandra grating spectra they find that most IPs are not compatible with the above model. Instead, they obtain a better fit with a photoionization code. This raises the issue of where the emission arises. One possibility is that the X-ray lines are predominantly from photoionized pre-shock mat crial. However. as discussed by Hellier \& Mukai (2004), Doppler shifts of the lines are of order $\sim 100 \mathrm{~km} \mathrm{~s}^{-1}$, rather than the $\sim 1000 \mathrm{~km} \mathrm{~s}^{-1}$ expected for material approaching the shock at near the escape velocity. Such low velocities imply an origin near the base of the accretion column where the material has been vastly decelerated (which is also expected in the standard model, since these are the densest regions and emission scales with density squared). But this leaves us with no coherent model for the spectral characteristics of the majority of IPs.

EX Hya is atypical, possibly owing to it being below the period gap and so having a much lower luminosity, which perhaps results in it being easier to model. Its importance will increase further now that we know its distance to high precision, given Beuermann et al.'s (2003) report of a parallax distance of $64.5 \pm 1.2 \mathrm{pc}$.

With EX Hya's parameters now securely known, Beuermann et al. report that the secondary is undermassive, being 18-30\% larger than a ZAMS star. Reassurringly, this corroborates the result of a large study of superhumps by Patterson et al. (2003), which shows that secondary stars in dwarf novae below the gap are $18 \%$ larger than ZAMS. From the relatively small scatter in the values of superhump period excess, Patterson et al. were also able to conclude that there is only one evolutionary track leading to these stars, most likely without any nuclear evolution of the secondaries.

\section{ARE THE SW SEX STARS MAGNETIC?}

The SW Sex phenomenon appears to be widespread in CVs (>20 systems show at least some SW Sex characteristics) and is present in at least one LMXB (Hynes et al. 2001). It is thus important for our understanding of accretion.

Nany recent authors have favored models which invoke magnetic fields to explain the SW Sex characteristics. regarding SW Sex stars as a variant of the IPs (e.g. Groot et al. 2001; Rodríguez-Gil et al. 2001; Hoard et al. 2003 and references therein; but see Hellier 2000 for a non-magnetic model). Such models are supported by suggestions of observed periodicities, including reports of periodic modulations in polarization data that, if verified, would clinch the magnetic nature of these stars.

However, at the risk of being thought unduely skeptical and ultimately proved wrong, I note that 
no periodicity has yet been corroborated by multiple datasets or by independent groups --- something that usually happens quickly for new IPs - and that CVs are notorious for flickering behavior that can mimic periodicities in limited datasets.

My main reason for skepticism about the magnetic nature of SW Sex stars is their general lack of X-ray emission, and particularly pulsed X-rays. IPs emit copious X-rays, with an obvious coherent pulsation. Polarization is much harder to find, and pulsed polarization has been seen in only a tenth of the known IPs.

In contrast, if the claims for SW Sex stars are true, their fields are strong enough to dominate the emission-line behavior, and to produce phase.variable polarization, but we do not see pulsed Xrays. This discrepancy, if true, would be telling us something fundamental about accretion.

A further implication concerns $\mathrm{VY} \mathrm{Scl}$ stars. There has been a long-standing problem over the lack of dwarf-nova outbursts in the low states of VY Scl stars, given that disk-instability models predict that they should occur. One idea is that irradiation keeps the inner disk too hot for such outbursts (Leach et al. 1999), but Hameury \& Lasota (2002) prefer a model in which a strong magnetic field evacuates the inner disk. They note that many SW Sex stars show VY Scl low states, and cite the magnetic models as support of their VY Scl hypothesis.

It is clear that the explanation of SW Sex behavior has wide implications. It would thus be good to have corroboration of periodicities sufficient to convince even an ardent skeptic, or to have sufficent null results to settle the matter the other way.

\section{THE WEIRD STARS FS AUR AND HS $2331+3905$}

As a last topic I turn to the stars FS Aur and HS $2331+3905$, although it is unclear whether the issue concerns magnetic fields or some sort of disk precession. FS Aur has an 86-min orbital period but also shows a large-amplitude photometric modulation at $3.4 \mathrm{hrs}$ (Tovmassian et al. 2003). No superhump has been seen with a period so much longer than the orbital period, but the period is also too short to be disk precession. Could it be the spin period of a magnetic white dwarf? Well, so far we know of no system with a spin period longer than the orbital period.
The issue becomes even stranger with the discrsery of HS 2331+3905 (Araujo-Betancor et al. 2004). This star has an 81-min orbital period and a 3.5-hr periodicity, making it similar to FS Aur. However. in FS Aur tho 3.t-hr periodicity appears in photometry only, and not in radial velocities, whereas in HS $2331+3905$ the 3.5 -hr periodicity is seen in radial velocities, but not in photometry. Explaining 3 -hr periodicities in 80-min binaries is hard enough without having to explain that also!

\section{REFERENCES}

Allan, A. et al. 1996, MNRAS, 279. 1345

Araujo-Betancor, S. et al. 2004, these proceedings

Beucrmann, K., Harrison, T. E., MicArthur, B. E.. Benedict, G.F., \& Gaensicke, B. T. 2003, A\&A, 412, 821

Cropper, M., Ramsay, 'G., Hellier, C., Mukai, K., Mauche, C., \& Pandel, D. 2002, Roy. Soc. Phil. Tr. A, 360, 1951

Evans, P. A., \& Hellier, C. 2004, in preparation

Evans, P. A.. Hellier, C., Ramsay, G., \& Cropper, M. 2004, MNRAS, in press

Groot, P. J., Rutten, R. G. M.. \& van Paradijs, J. 2001. A\&A, 368,183

Haberl, F., \& Motch, C. 1995, A\&A, 297, L37

Hameury, J.-M., \& Lasota, J.-P. 2002, A\&A, 394. 231

Hellier, C., 1995, in ASP Conf. Ser., 85. "Cape Workshop on Magnetic Cataclysmic Variables", eds. Buckley, D., Warner, B. (San Francisco: ASP), 185

Hellier, C., 2000, New Ast. Rev., 44, 131

Hellier, C., Harmer, S., \& Beardmore, A. P. 2(0)4. MINRAS, in press.

Hellier, C., \& Mukai, K. 2004, MNRAS, submitted

Hoard, D. W., Szkody, P., Froning, ('. S., Long, k. S.. \& Knigge, C. 2003, AJ, 126, 2473

Hynes, R. I. et al. 2001, MNRAS, 324, 180

Leach, R., Hessman, F. V. King, A. R., Stehle. R., \& Mrattei, J. 1999, MNRAS, 305, 225

Mauche, C. W. 2002, ApJ, 580. 12:3

Mason, K. O. 1997, MNRAS, 285. 19:3

Mukai, K., Kinkhabwala, A., Peterson, J.R., Kahn, S. M., \& Paerels, F. 2003, ApJ, 586, Li7

Patterson, J. et al. 2003, PASP, 115, 1308

Ramsay, G., \& Cropper, M. 2004, NINRAS. 3.7T. 497

Rodríguez-Gil, P., Casares, J., Nartinez-Pais, I. G.. Hakala, P., \& Steeghs, D., 2001, ApJ, 548. L49

Tovmassian, G. et al. 2003, PASP, 115, 725

Warner, B., \& Woudt, P. 2002, MNRAS. 335. \&4

Warner, B., Woudt, P., \& Pretorius, M. L. 2003, MINR.AS, 344,1193

Woudt, P., \& Warner, B. 2002, MNRAS. 333, 411

Coel Hellier: Keele University. Staffordshire, ST5 5BG, U.K. (ch'(1astro.keele.ac.uk). 